\title{
HIGHLIGHT
}

\section{Gene-editing in plants no longer requires tissue culture}

\author{
Yi ZHANG ${ }^{1}$, Michael PALMGREN (凶) ${ }^{2}$ \\ 1 Copenhagen Plant Science Centre, Department of Plant and Environmental Sciences, University of Copenhagen, Thorvaldsensvej 40, \\ 1871 Frederiksberg C, Denmark \\ 2 NovoCrops Center, Department of Plant and Environmental Sciences, University of Copenhagen, Thorvaldsensvej 40, \\ 1871 Frederiksberg C, Denmark
}

Plant gene editing, which can produce targeted modifications in plants, shows great potential for gene function analysis and precision breeding of crops $^{[1]}$. To produce gene-edited plants, gene editing reagents ${ }^{[2]}$ (for example, CRISPR/Cas9 components) need to be delivered to plant cells. This involves a lengthy, costly and labor-intensive tissue culture step, which, moreover, is currently only possible in limited number of plant species, making it a major bottleneck in plant gene editing. In the recent issue of Nature Biotechnology, a research team from the University of Minnesota led by Daniel F. Voytas, describes a new method that produces gene-edited plants while sidestepping the need for tissue culture (Fig. 1 $)^{[3]}$. The method takes advantage of the de novo induction of meristems.

Differentiated plant cells generally cannot divide or produce cells of a different type. However, previous studies have shown that already differentiated cells can be induced to form meristems by ectopic (i.e., out-of-place) expression of specific developmental regulators. Meristems are plant tissues that include undifferentiated stem cells (meristematic cells) capable of cell division and able to give rise to various tissues and organs. For example, in Arabidopsis, the WUSCHEL (WUS) gene plays a key role in embryogenesis, and overexpressing WUS can promote the vegetative-to-embryonic transition ${ }^{[4]}$. The combined ectopic expression of SHOOT MERISTEMLESS (STM) and WUS activates a subset of meristem functions in Arabidopsis, including cell division and organogenesis ${ }^{[5]}$. The ipt gene, located on the Ti-plasmid of the soil bacterium Agrobacterium tumefaciens, encodes the enzyme isopentenyl transferase, which in plants induces the biosynthesis of cytokinines to stimulate organogenesis ${ }^{[6]}$. In monocots, overexpression of the Baby boom $(\mathrm{Bbm})$ and WUS genes increases transformation efficiency by promoting the formation of embryos from somatic cells ${ }^{[7]}$.

The group of Voytas hypothesized that meristems could be induced by the help of developmental regulators. To test this idea, maize WUS2, A. thaliana STM, A. tumaefaciens ipt and other developmental regulators were expressed in Nicotiana benthamiana plants in different combinations using a variety of promoters. Agrobacterium was used to deliver the transgenes and luciferase was used as a reporter. Meristem-like structures were formed that grew into transgenic plants with luciferase expression and the trait was found to be heritable.Then, using the same method, a single guide RNA (sgRNA) targeting two test genes was introduced together with the successful combination of developmental regulators into leaves of transgenic $N$. benthamiana constitutively expressing Cas9. In the shoots generated, editing of the target genes could be verified and the mutations were found to be transmitted to the next generation. The question then arose as to whether meristems could also be induced on soil-grown plants. This approach indeed proved successful in a number of dicot species, besides $N$. benthamiana also in potato and grapevine. Moreover, geneedited $N$. benthamiana plants were generated, and some edited plants were found not to harbor the transgenes used for editing.

The de novo meristem induction method, which was termed Fast-TrACC (fast-treated Agrobacterium co-culture), has clear advantages over traditional tissue culture procedures (Fig. 1). First, it drastically reduces the time needed for producing gene-edited plants from several months to a few weeks. Second, Fast-TrACC does not require sterile conditions and works with plants that have been grown in soil. The tissue culture method demands the use of a sterile bench and sterile media, so the tissue culture-free method requires fewer resources and is feasible for smaller groups. Third, when Cas9 was delivered together with the sgRNA, gene-edited plants were in some cases produced

Received February 7, 2020

Correspondence: palmgren@plen.ku.dk

(C) The Author(s) 2020. Published by Higher Education Press. This is an open access article under the CC BY license (http://creativecommons.org/licenses/by/4.0) 


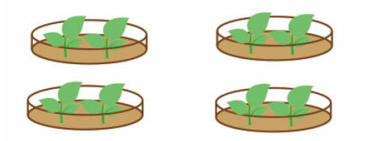

Seedlings from liquid culture

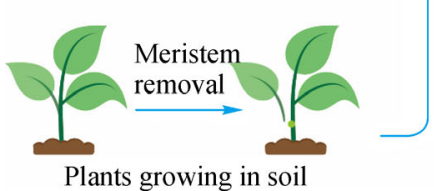

Plants growing in soil
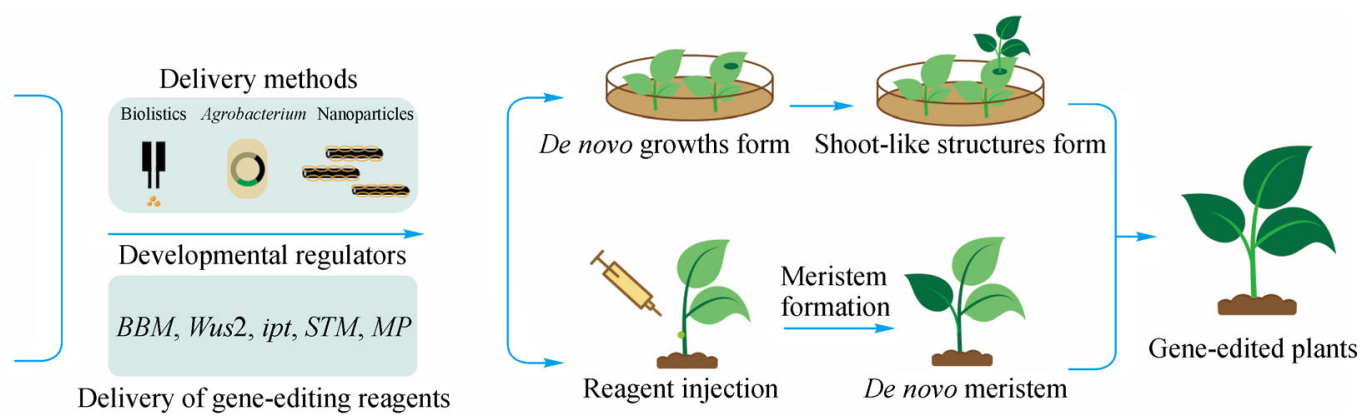

Fig. 1 Gene-edited plants produced by de novo meristem induction. Optimal developmental regulators combinations and gene editing reagents (e.g., CRISPR/Cas9 components) are delivered to somatic plant cells by Agrobacterium, biolistics or nanoparticles. Then de novo gene-edited meristems are induced and gene-edited shoots produced.

that lacked integrated transgenes (Fig. 1). With the traditional tissue culture method, once gene-edited plants have been created, a selfing or crossing step is needed to segregate away the gene editing reagents, which are transgenes and may continue to be expressed and can cause off-target effects ${ }^{[8]}$. Finally, Fast-TrACC is applicable in a wider variety of plant species, which substantially broadens the utility of genome editing in plants.

Nevertheless, the de novo meristem induction method may have its limitations. The Voytas group only used Agrobacterium-mediated delivery, which does not work for all plants, but other means of delivery, including biolistics and nanoparticles, could be employed (Fig. 1). A number of transgenic plants resulting from Fast-TrACC exhibited developmental abnormalities such as curled leaves, probably because of persistent expression of developmental regulators, which could complicate the assigning function to genes that have been edited and, in particular, the method may not be suitable for testing the function of genes involved in plant development. Further, some mutants produced by the method were found to be chimeric, i.e., several mutant types exist in the same plant, creating problems for subsequent experimental analysis.

The Fast-TrACC method greatly simplifies the task of creating gene-edited plants. Since tedious tissue culture procedures can now be avoided, gene editing can proceed much faster and may work on a much wider variety of plants. This successful proof of concept is a major contribution towards the coming era of precision crop breeding.

Acknowledgements We thank Yanpeng Wang (Institute of Genetics and Developmental Biology, Chinese Academy of Sciences, Beijing, China) for help with the figure. Yi Zhang was supported by the Innovation Fund Denmark grant 8055-00038A, Michael Palmgren by the Novo Nordisk Foundation Challenge grant NNF19OC005658.

Compliance with ethics guidelines Yi Zhang and Michael Palmgren declare that they have no conflicts of interest or financial conflicts to disclose.

This article is a highlight and does not contain any studies with human or animal subjects performed by any of the authors.

\section{References}

1. Chen K, Wang Y, Zhang R, Zhang H, Gao C. CRISPR/Cas genome editing and precision plant breeding in agriculture. Annual Review of Plant Biology, 2019, 70: 667-697

2. Ran Y, Liang Z, Gao C. Current and future editing reagent delivery systems for plant genome editing. Science China Life Sciences, 2017, 60(5): 490-505

3. Maher M F, Nasti R A, Vollbrecht M, Starker C G, Clark M D, Voytas D F. Plant gene editing through de novo induction of meristems. Nature Biotechnology, 2020, 38: 84-89

4. Zuo J, Niu Q W, Frugis G, Chua N H. The WUSCHEL gene promotes vegetative-to-embryonic transition in Arabidopsis. Plant Journal, 2002,30 (3): 349-359

5. Gallois J L, Woodward C, Reddy G V, Sablowski R. Combined SHOOT MERISTEMLESS and WUSCHEL trigger ectopic organogenesis in Arabidopsis. Development, 2002, 129(13): 3207-3217

6. Smigocki A C, Owens L D. Cytokinin gene fused with a strong promoter enhances shoot organogenesis and zeatin levels in transformed plant cells. Proceedings of the National Academy of Sciences of the United States of America, 1988, 85(14): 5131-5135

7. Lowe K, Wu E, Wang N, Hoerster G, Hastings C, Cho M J, Scelonge C, Lenderts B, Chamberlin M, Cushatt J, Wang L J, Ryan L, Khan T, Chow-Yiu J, Hua W, Yu M, Banh J, Bao Z M, Brink K, Igo E, Rudrappa B, Shamseer P M, Bruce W, Newman L, Shen B, Zheng P Z, Bidney D, Falco C, Register J, Zhao Z Y, Xu D P, Jones T, Gordon-Kamm W. Morphogenic regulators Baby boom and Wuschel improve monocot transformation. Plant Cell, 2016, 28: 1998-2015

8. Zhang Y, Gao C. Recent advances in DNA-free editing and precise base editing in plants. Emerging Topics in Life Sciences, 2017, 1(2): 161-168 